\title{
Estimulação encefálica profunda - Uma atualização
}

\author{
Eddy Krueger', Edwing Martin Holguin Wilson², Percy Nohama ${ }^{3}$ \\ Laboratório de Engenharia de Reabilitação (LER) da Universidade Tecnológica Federal do Paraná (UTFPR), Campus \\ Curitiba, PR.
}

\section{RESUMO}

A aplicação de estímulos elétricos nas regiões profundas do cérebro é definida como estimulação encefálica profunda (EEP). A EEP pode ser aplicada em patologias motoras e psicológicas, reduzindo ou tornando desnecessária a administração de medicação complementar. O objetivo deste artigo é mostrar uma atualização pertinente à EEP, baseando-se principalmente em artigos do último triênio. Foram selecionados artigos das bases de pesquisa Wiley Online Library (3), ScienceDirect (8), SciELO (1), BioMed Central (1), Google acadêmico (17) e livros (7), totalizando 37 referências selecionadas, das quais 15 artigos foram publicados no ano de 2010 e três, publicados em 2011. Dentre os parâmetros estimulatórios mais empregados durante o triênio 2009 a 2011, destacam-se: amplitude de 1 a $10 \mathrm{~V}$; corrente entre 50 e $300 \mu A$; frequência de 130 a $185 \mathrm{~Hz}$ e período ativo do pulso entre 60 e $220 \mu \mathrm{s}$. A EEP mostra-se como recurso viável para tratamento de patologias que apresentam distúrbios de movimento, como doença de Parkinson, distonias e mioclonias. Além disso, a EEP é eficiente para o tratamento de distúrbios psicológicos/neurológicos como depressão, epilepsia e cefaleia e possui forte incentivo a pesquisas que tenham aplicações diferenciadas, como o tratamento de viciados em drogas.

\section{PALAVRAS-CHAVE}

Estimulação encefálica profunda, córtex cerebral/cirurgia, revisão.

\section{ABSTRACT}

Deep brain stimulation - An update

The application of electrical stimuli in deep brain areas is defined as deep brain stimulation (DBS). $D B S$ can be applied to motor and psychological diseases, reducing or obviating the administration of additional medication. The aim of this paper is to present an update about DBS, based primarily on articles published on the last three years. There were selected articles from the databases Wiley Online Library (3), ScienceDirect (8), SciELO (1), BioMed Central (1), Google Scholar (17) and books (7), totaling 37 references, where, 15 articles were published in 2010 and three published in 2011. Among the main stimulatory parameters employed during the triennium 2009 to 2011 are: amplitude from 1 to $10 \mathrm{~V}$; current from 50 to $300 \mu \mathrm{A}$; frequency from 130 to $185 \mathrm{~Hz}$ and pulse active period between 60 and $220 \mu \mathrm{s}$. DBS proves to be a viable resource for treatment of diseases that have movement disorders such as Parkinson's disease, dystonia and myoclonus. In addition, the DBS is effective for the treatment of psychological/neurological disorders, such as depression, epilepsy, headache, and has a strong research incentive to applications as treatment of drug addicts.

\section{KEY-WORDS}

Deep brain stimulation, cerebral cortex/surgery, review.

1. Fisioterapeuta, MSc. em Engenharia Biomédica pela Universidade Tecnológica Federal do Paraná (UTFPR), doutorando em Engenharia Biomédica (Programa de Pós-Graduação em Engenharia Elétrica e Informática Industrial - CPGEI) pela UTFPR, Curitiba, PR, Brasil.

2. Médico, MSc. em Saúde Coletiva pela Universidade Luterana do Brasil (ULBRA-RS), especialista em Toxicologia Clínica, Epidemiologia e Neuroanatomia, Instituto de Cardiologia do Rio Grande do Sul, Porto Alegre, RS, Brasil.

3. Doutor em Engenharia Biomédica pela Universidade Estadual de Campinas (Unicamp), Campinas, SP, docente do Programa de Pós-Graduação em Tecnologia em Saúde (PPGTS) na Pontifícia Universidade Católica do Paraná (PUCPR) e do Departamento Acadêmico de Eletrônica (DAELN/CPGEI) na UTFPR, Curitiba, PR, Brasil. 


\section{Introdução}

Os neurônios são estruturas suscetíveis a variações no potencial elétrico de sua membrana celular quando expostos a um campo elétrico variável. ${ }^{28} \mathrm{~A}$ estimulação encefálica profunda (EEP) ativa estruturas cerebrais por meio de eletrodos implantados, para tratamento de patologias neurológicas e psicológicas, ${ }^{10,17}$ reduzindo ou tornando desnecessária a administração de medicação. ${ }^{10} \mathrm{~A}$ EEP é aplicada principalmente nos gânglios (ou núcleos) da base, ${ }^{16}$ como a estimulação em globo pálido e núcleo subtalâmico, ${ }^{23}$ tálamo, ${ }^{12}$ entre outros. As patologias tratadas com EEP são voltadas para distúrbios de movimento como: doença de Parkinson, distonia, tremor de ação, ${ }^{20}$ síndrome de Tourette, ${ }^{1}$ ou em transtornos neurológicos, como a estimulação da área branca da região subcalosa do giro cingulado (área 25 de Korbinian Brodmann (1868-1918) para depressão. ${ }^{15}$ Mesmo aprovada como viável para alguns tratamentos, a EEP pode ocasionar efeitos adverso ${ }^{32} \mathrm{em}$ alguns pacientes, como morte em alguns casos, devido ao procedimento cirúrgico. ${ }^{38}$

Desse modo, a EEP vem se tornando uma técnica cada vez mais difundida; porém encontra-se em evolução. Este artigo tem por objetivo apresentar o estado da arte da aplicação da EEP, abordando patologias aplicadas, regiões estimuladas e principais parâmetros utilizados.

\section{Método}

A busca de informações foi realizada nas bases Wiley Online Library, ScienceDirect, SciELO, Biomed Central, Google acadêmico e livros referentes ao assunto. O idioma de preferência selecionado foi o inglês, com as palavras-chave: "deep brain stimulation" e "stereotactic neurosurgery". Efetuou-se a procura de artigos com uma janela de tempo de 1990 a 2011 e livros de 2003 a 2008. Foram descartados os trabalhos que não estavam de acordo com o escopo do artigo. Após a realização da busca nas bases de dados, foram lidos os abstracts e eliminadas as duplicações. Dos trabalhos selecionados, foram extraídas informações a respeito dos seguintes tópicos: estimulação encefálica profunda e cirurgia por estereotaxia.

\section{Resultados}

Todos os trabalhos que foram selecionados versavam sobre estimulação encefálica profunda. A síntese elaborada baseou-se em de três artigos da Wiley Online Library (um havia sido excluído), oito da ScienceDirect, um da SciELO, um da Biomed Central, 17 do Google acadêmico (dois haviam sido excluídos) e trechos de oito livros (um foi excluído posteriormente) totalizando 37 referências. A tabela 1 mostra o número de artigos e livros utilizados no estudo, divididos por ano.

Tabela 1 - Número de artigos e livros utilizados e seus respectivos anos de publicação

\begin{tabular}{lcc}
\hline & \multicolumn{2}{c}{ Número de obras } \\
\cline { 2 - 3 } Ano & Artigo & Livro \\
\hline 1988 & 1 & \\
1990 & 1 & \\
1999 & 1 & 1 \\
2001 & & 1 \\
2003 & & 2 \\
2005 & 1 & 1 \\
2006 & & 1 \\
2007 & & 1 \\
2008 & 1 & \\
2009 & 6 & \\
2010 & 17 & \\
2011 & 3 & \\
Total & 31 & \\
\hline
\end{tabular}

\section{Estimulação encefálica profunda}

Eletrodos implantáveis intracerebrais são colocados por processo de estereotaxia (desenvolvida em 1918), ${ }^{33}$ geralmente guiada por imagem de ressonância magnética, utilizada em cirurgias de remoção de foco epileptogênico. ${ }^{26}$ Antes do advento da EEP, Meyer afirmou, em 1939, que lesões cirúrgicas nos gânglios da base ocasionavam alívio em desordens de movimento, como mostra a figura 1. Como a cirurgia tinha de ser exposta, o índice de mortes por consequência do procedimento cirúrgico era alto (aproximadamente 14\%). ${ }^{36}$

A estereotaxia funcional era utilizada no decênio de 1940 para cirurgias de lobotomia, reduzindo, assim, a agressão cirúrgica e consequências diretas. ${ }^{20} \mathrm{Na}$ década de 1970, realizou-se o primeiro implante de EEP. ${ }^{36}$ Atualmente, as cirurgias de implante e EEP utilizam a estereotaxia com o auxílio de técnicas de imagens 3-D, ${ }^{32}$ como mostra a figura 2.

O eletrodo mais utilizado na EEP é o quadripolar, ${ }^{10}$ como o ilustrado na figura 3, e nele se pode selecionar qual dos quatro contatos será o eletrodo ativo. $\mathrm{O}$ formato da onda aplicada é quadrático, podendo ser monofásico 
ou bifásico, ${ }^{10}$ como ilustra a figura 4 , com estimulação anódica ou catódica dependendo do eletrodo ativo. O implante do estimulador elétrico pode ocorrer na região do tórax (Figura 5), assim como pode ser colocado na região subclavicular ${ }^{4}$ ou diretamente no crânio ${ }^{10}$ (Figura 6).

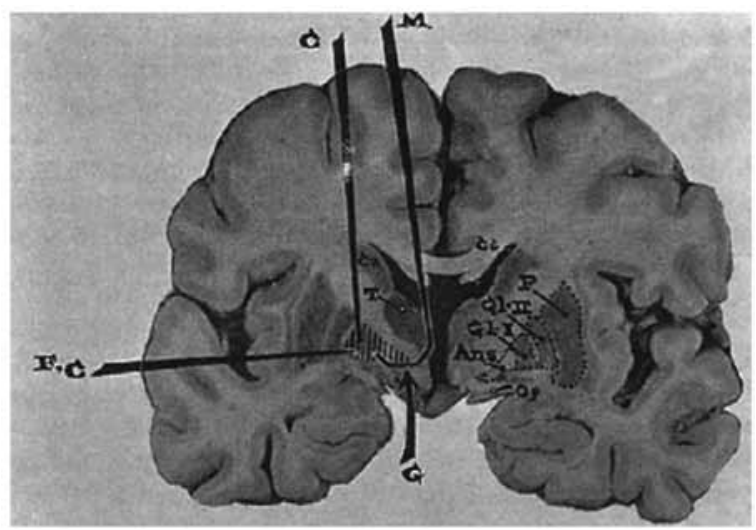

Figura 1 - Lesão cirúrgica sobre os gânglios da base para tratamento de desordens de movimento utilizada em 1939. Retirada de Schwalb e Hamani (2008). ${ }^{36}$

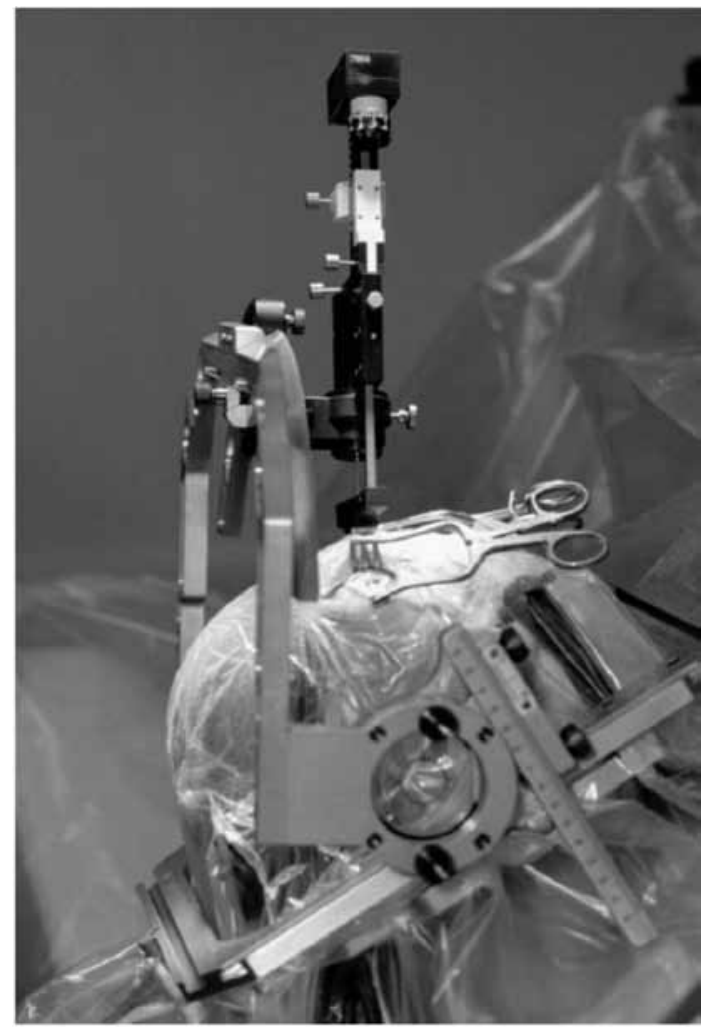

Figura 2 - Foto de cirurgia por estereotaxia. Extraída de Pinault (2010). ${ }^{32}$
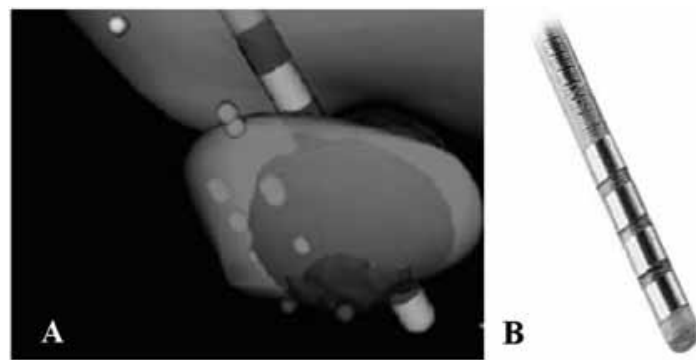

Figura 3 - (A) Ativação tecidual do núcleo subtalâmico em diferentes regióes com eletrodo quadripolar. (B) Eletrodo quadripolar. Retirada de Alberts et al. $(2010)^{3}$ e de Wojtecki et al. (2011). ${ }^{39}$

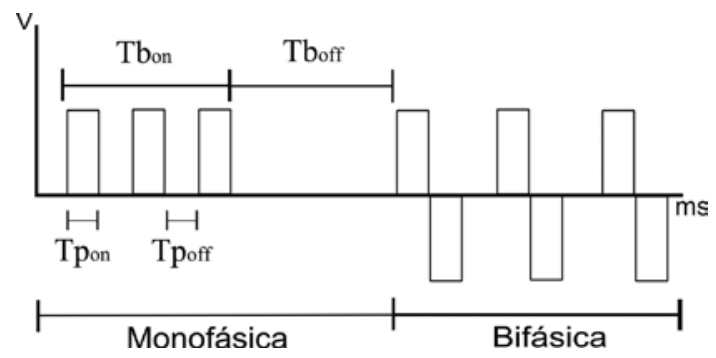

Figura 4 - Duração de pulso com onda quadrada e de burst. Tempos de alta $\left(T p_{o n}\right)$ e de baixa do pulso $\left(T p_{o f}\right)(e m \mu s)$ informam a frequência da portadora. On $e$ off $\mathrm{do}$ burst (em $\mathrm{ms}$ ) informam a duração do burst. Monofásica - pulso em apenas uma polaridade (positiva ou negativa); Bifásica - pulso alterna a sua polaridade a cada pulso. $T b_{\text {on }}$ : período ativo do burst; $T b_{o f f}$ : período inativo do burst; $T p_{o n}$ : período ativo do pulso; $T p_{o f f}$ período de repouso do pulso. Retirada de Krueger-Beck et al. (2011). ${ }^{21}$

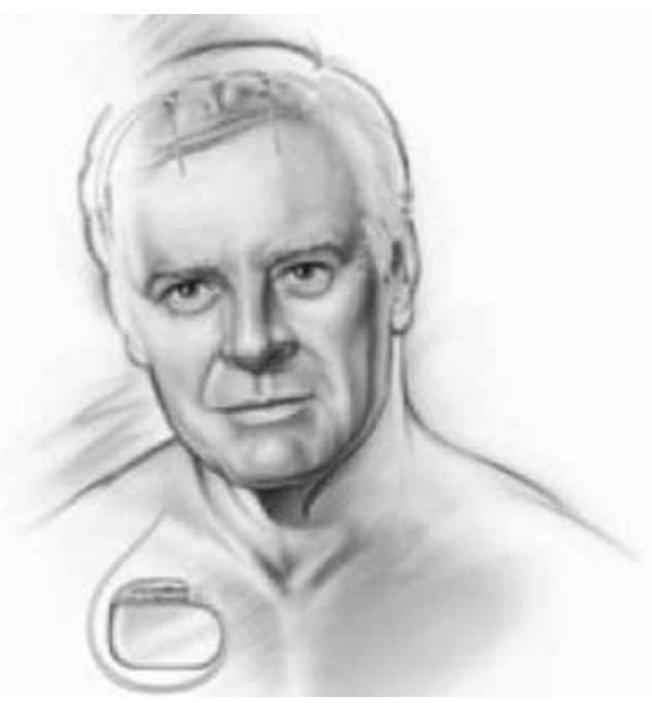

Figura 5 - Estimulador elétrico para estimulação encefálica profunda implantado no tórax. Retirada de Schwalb e Hamani (2008). ${ }^{36}$ 


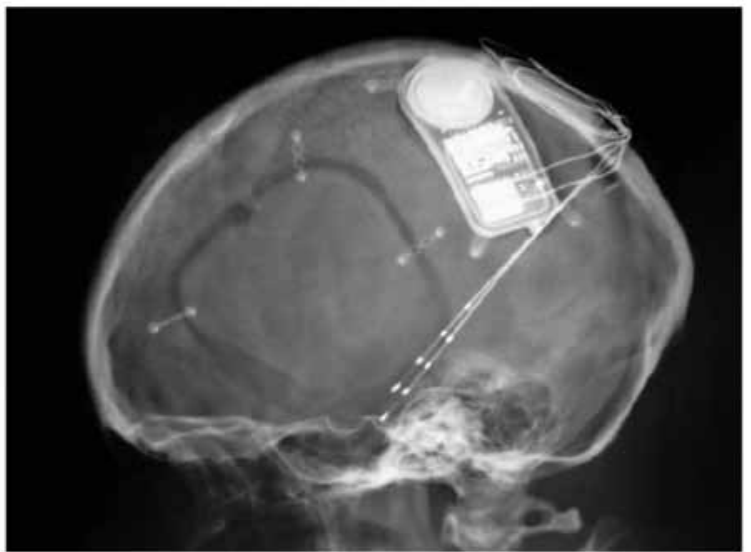

Figura 6 - Implante do estimulador elétrico no crânio. Retirada de DiLorenzo e Bronzino (2007). ${ }^{10}$

No Brasil, o primeiro procedimento destinado ao tratamento da epilepsia envolvendo estimulação elétrica no córtex cerebral foi iniciado no ano de 1974 pelo Prof. Raul Marino Júnior. ${ }^{34} \mathrm{O}$ Prof. Manuel Jacobsen Teixeira, além de ter sido o primeiro a utilizar a EEP na substância periaquedutal mesencefálica destinada ao tratamento da dor no ano de 1982, foi um dos primeiros a utilizar a técnica de estimulação encefálica profunda para tratar distúrbios de movimento. Recentemente, o Prof. Manuel Jacobsen Teixeira e sua equipe realizaram a primeira cirurgia para implantação de marca-passo cerebral recarregável no Brasil, tornando desnecessários novos procedimentos cirúrgicos para substituição de baterias. ${ }^{18}$

A EEP é aplicada não somente em transtornos motores, mas também psiquiátricos, como no tratamento de transtornos de desordem obsessivo-compulsiva, ${ }^{22}$ depressão ${ }^{15}$ e ansiedade. ${ }^{7}$ Em núcleos profundos do tálamo, a EEP serve para tratamentos somatossensoriais, como de dor crônica, paralisia cerebral, epilepsia e espasticidade. ${ }^{36}$ Há também indicações da EEP para casos de pacientes com desordens de consciência, tal como estado vegetativo ou de consciência mínima. ${ }^{37}$ Rouaud et al. ${ }^{35}$ (2010) avaliaram a EEP bilateral do núcleo subtalâmico na redução do desejo de consumir cocaína em ratos $(\mathrm{N}=88)$. Os testes indicaram que a EEP reduziu a vontade de consumir cocaína injetável, entretanto aumentou o desejo por alimentos com sacarose.

A patologia com uma das maiores aplicações da EEP é a doença de Parkinson, ${ }^{3,25,30,31,38}$ que ocorre devido à degeneração dos neurônios dopaminérgicos da substância negra compacta, ocasionando alterações cognitivas $^{9}$ e de movimento como tremor. ${ }^{27}$ Segundo Krack et al. (2010), ${ }^{20}$ as frequências utilizadas para redução do tremor de Parkinson estão entre 50 e 200 Hz. Em um artigo de revisão de literatura, Benabid et al. $(2009)^{5}$ mostraram que, para a EEP com estimulação monopolar do núcleo subtalâmico para mal de Parkinson, os parâmetros utilizados foram: estimulação com amplitude de 2,9 $\pm 0,6 \mathrm{~V}$, frequência de $139 \pm 18 \mathrm{~Hz}$, período ativo do pulso em $63 \pm 7,7 \mu$ s. A estimulação de núcleos pedúnculo-pontinos com EEP vem sendo realizada em modelos animais, visando ao tratamento de desordens de movimento decorrentes da doença de Parkinson e instabilidade postural. ${ }^{2}$

A tabela 2 lista os parâmetros estimulatórios utilizados e as doenças tratadas por alguns autores nos anos de 2009 e 2011. As figuras 7 e 8 complementam a tabela 2, ilustrando o posicionamento dos eletrodos no encéfalo com cortes distintos do encéfalo, transversal e sagital respectivamente, mostrando os pontos de EEP utilizados pelos autores no triênio 2009-2011.

Borggraefe et al. ${ }^{8}$ (2010) estudaram a aplicação de EEP em distonia generalizada primária. Participaram da pesquisa seis adolescentes, nos quais ocorreu estimulação no globo pálido interno bilateralmente. Por intermédio da correlação dos testes pré e pós-cirurgia, os resultados foram favoráveis à EEP.

Gutman et al. ${ }^{15}$ (2009) avaliaram qual ponto de estimulação no encéfalo tinha a melhor resposta para a depressão. As áreas de aplicação abrangeram: (A) a área branca da região subcalosa do giro cingulado e (B) o braço anterior da cápsula interna. Os resultados foram avaliados com técnica de imagem por ressonância magnética com tensor de difusão. A região A mostrou uma conexão ipsilateral consistente com $(1 \mathrm{~A})$ o córtex medial frontal, $(2 \mathrm{~A})$ a extensão anteroposterior do giro do cíngulo, $(3 \mathrm{~A})$ o lobo temporal médio, $(4 \mathrm{~A})$ a região dorso medial do tálamo, (5A) o hipotálamo, (6A) o núcleo accumbens e $(7 \mathrm{~A})$ o tronco cerebral. A região $\mathrm{B}$ mostrou projeções para o (1B) polo do lobo frontal, (2B) o lobo temporal medial, (3B) o cerebelo, (4B) a região dorsal do tálamo, (5B) o hipotálamo, (6B) o núcleo accumbens e (7B) o tronco cerebral. As áreas de conexão comuns às duas regiões estimuladas abrangeram: ( $1 \mathrm{AB})$ o polo do lobo frontal, (2AB) o lobo temporal medial, $(3 \mathrm{AB})$ o núcleo accumbens, $(4 \mathrm{AB})$ a região dorsal do tálamo e (5AB) o hipotálamo. Os pesquisadores concluíram que essas regiões sugerem redes neurais amplas, com áreas estimuladas envolvidas na resposta de ativação da depressão e outras áreas envolvidas na inibição da depressão.

Okun et al..$^{30}$ (2009) estudaram a efetividade de diferentes regiões estimuladas com a EEP no tratamento da doença de Parkinson. Foram avaliados durante sete meses, após a intervenção cirúrgica, pacientes $(\mathrm{N}=45)$ com implantes randomizados, divididos em estimulação da lâmina interna do globo pálido $(\mathrm{N}=$ 23) e estimulação no núcleo subtalâmico $(\mathrm{N}=22)$. As estimulações foram deflagradas unilateralmente. As avaliações incluíram teste de humor e felicidade. Resultados mostraram que não houve diferença da EEP entre o núcleo subtalâmico e a lâmina interna do globo pálido para a doença de Parkinson. 


\begin{tabular}{|c|c|c|c|c|}
\hline \multirow[t]{2}{*}{ Autores } & \multicolumn{3}{|c|}{ Parâmetros estimulatórios } & \multirow[t]{2}{*}{ Patologia/Distúrbio } \\
\hline & Tensão (V) & Frequência (Hz) & $\begin{array}{l}\text { Período ativo } \\
\text { do pulso }(\mu \mathrm{s})\end{array}$ & \\
\hline Rouaud et al. $(2010)^{35}$ & $50-130^{5}$ & 130 & 60 & Desejo de utilizar cocaína \\
\hline Gutman et al. (2009) ${ }^{15}$ & * & * & * & Depressão \\
\hline Borggraefe et al. $(2010)^{8}$ & * & * & * & Distonia generalizada primária \\
\hline Okun et al. $(2009)^{30}$ & 2,1 a 3,3 & 129,2 a 170,8 & 71,8 a 99,4 & Doença de Parkinson \\
\hline Fisher et al. $(2010)^{12}$ & 5 & 145 & 90 & Epilepsia \\
\hline Weaver et al. $(2009)^{38}$ & * & * & * & Doença de Parkinson \\
\hline Follett et al. $(2010)^{13}$ & GPI: 3,9 NST: 3,1 & GPI: 168 NST: 165 & GPI: 95,7 NST: 75,9 & Doença de Parkinson \\
\hline Bewernick et al. $(2010)^{7}$ & 2 a 10 & 130 a 150 & 90 a 220 & Depressão e anedonia \\
\hline Maks et al. $(2009)^{25}$ & 1,5 a 3,6 & 130 a 185 & 60 a 90 & Doença de Parkinson \\
\hline Péron et al. $(2010)^{31}$ & $\begin{array}{l}\text { D: } 2,6 \pm 0,6 \\
\text { E: } 2,5 \pm 0,4\end{array}$ & $\begin{array}{l}\text { D: } 140,7 \pm 17,4 \\
\text { E: } 141,4 \pm 17,2\end{array}$ & $\begin{array}{c}\text { D: } 65,7 \pm 12 \\
\text { E: } 64,2 \pm 10,7\end{array}$ & Doença de Parkinson \\
\hline Alberts et al. $(2010)^{3}$ & 2 a 3,2 & 130 a 185 & 60 & Doença de Parkinson \\
\hline Fontaine et al. $(2010)^{14}$ & $3(\max )$ & 185 & 60 & Cefaleia em salva \\
\hline Ackermans et al. $(2010)^{1}$ & 1 a 8 & 100 a 130 & 90 a 210 & Síndrome de Tourette \\
\hline Azoulay-Zyss et al. (2011) & 1,6 a 4,7 & 130 & 60 & Mioclonia-Distonia \\
\hline Klein et al. $(2011)^{19}$ & $300^{5}$ & 130 & * & Modelo animal \\
\hline Wojtecki et al. $(2011)^{39}$ & 3,5 & 125 & 60 & Doença de Parkinson \\
\hline
\end{tabular}

§: valor expresso em corrente elétrica $(\mu \mathrm{A})$; GPI: globo pálido interno; NST: núcleo subtalâmico; D: lado direito, E: lado esquerdo; Max: valor máximo; *: dado não apresentado.

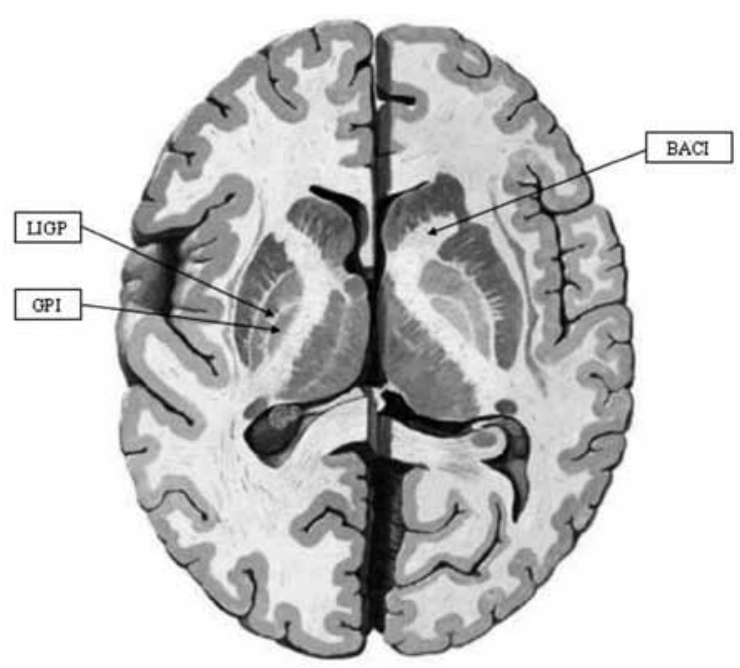

Figura 7 - Corte transversal do encéfalo. LICP: lâmina interna do globo pálido, ${ }^{30}$ GPI: globo pálido interno, ${ }^{4,8,13,38}$ BACI: braço anterior da cápsula interna. ${ }^{15}$ Modificada de Felten e Józefowicz $(2005)^{11}$ e Netter (2001). ${ }^{29}$

Fisher et al. ${ }^{12}$ (2010) avaliaram a estimulação bilateral do núcleo anterior do tálamo para epilepsia. Um mês após da intervenção cirúrgica, o estudo dividiu-se em duas etapas. Durante (1) três meses de aplicação, foi

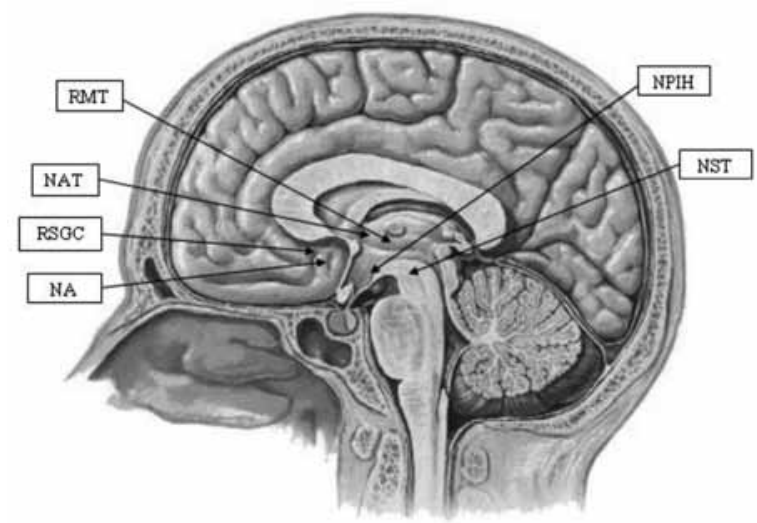

Figura 8 - Corte sagital do encéfalo. RMT: região medial do tálamo; ${ }^{1}$ NAT: núcleo anterior do tálamo, ${ }^{12}$ RSGC: região subcalosa do giro do cíngulo, ${ }^{15} \mathrm{NA}$ : núcleo acumbens ${ }^{7}$, NPIH: núcleo posteroinferior do hipotálamo, ${ }^{14}$ NST: núcleo subtalâmico. ${ }^{3,13,25,30,31,38,39}$ Modificada de Felten e Józefowicz $(2005)^{11} e$ Netter (2001). ${ }^{29}$

realizado um estudo duplo-cego, em que um grupo $(\mathrm{A})$ recebia estimulação $(\mathrm{N}=55)$ e outro grupo $(\mathrm{B})$ não $(\mathrm{N}=$ 55). Nesses três meses, o grupo A recebia a aplicação de $5 \mathrm{~V}$ contra $0 \mathrm{~V}$ do grupo B. A estimulação era aplicada 
constantemente durante 1 min e permanecia desligada durante $5 \mathrm{~min}$. A média de ataques epilépticos ao mês era de 19,5 para todos os participantes. Ao final dos três meses, o grupo que recebeu estímulo obteve $29 \%$ de redução no número de ataques em comparação com o grupo controle. Após essa etapa, o grupo B que teve a aplicação falsa (placebo) começou a ser estimulado, iniciando uma nova etapa (2) que durou dois anos (N =110). Ao final dos dois anos, houve 56\% de redução média da frequência das crises, $54 \%$ dos pacientes tiveram redução de ataques epilépticos de pelo menos 50\% (comparando ao estado pré-EEP) e 14 pacientes permaneceram livres de crises epilépticas durante os últimos seis meses do estudo. Cinco óbitos foram registrados devido a processo cirúrgico, mas nenhum decorrente da implantação ou do uso da EEP.

Weaver et al. ${ }^{38}$ (2009) compararam a EEP com a terapia médica tradicional para o tratamento da doença de Parkinson durante seis meses. Foram divididos três grupos em: (1) EEP bilateral no núcleo subtalâmico $(\mathrm{N}=$ 60), (2) EEP bilateral no globo pálido $(\mathrm{N}=61)$ e (3) com terapia médica tradicional $(\mathrm{N}=134)$. As aplicações de EEP perduraram 4,6 horas diárias. Surgiram 39 efeitos adversos decorrentes das intervenções cirúrgicas e uma morte por hemorragia. Os resultados mostraram que a EEP foi mais eficiente que a terapia médica tradicional, melhorando a condição motora e a qualidade de vida dos participantes durante os seis meses avaliados.

Com o mesmo objetivo, Follett et al. ${ }^{13}$ (2010) compararam as diferenças da EEP bilateral entre o núcleo subtalâmico e o globo pálido interno no tratamento da doença de Parkinson. Foram avaliados os 24 meses iniciais do tratamento de pacientes com estimulação no núcleo subtalâmico $(\mathrm{N}=147)$ e no globo pálido interno $(\mathrm{N}=152)$. A média dos resultados não mostrou significância estatística entre eles $(\mathrm{p}=0,50)$. Pacientes com estimulação do núcleo subtalâmico necessitavam de dose menor de agentes dopaminérgicos comparados com os pacientes com EEP no globo pálido interno $(\mathrm{p}=0,02)$. Entretanto, a capacidade de movimentar-se rapidamente após um estímulo visual foi pior no grupo do núcleo subtalâmico $(\mathrm{p}=0,03)$. O nível do quadro depressivo apresentou piora após a cirurgia em pacientes com EEP no núcleo subtalâmico e melhora do quadro em pacientes com estimulação do globo pálido interno $(\mathrm{p}=0,02)$.

A EEP pode ter a capacidade de inibir a função do núcleo em vez de excitá-lo. Bergman et al. ${ }^{6}$ (1990) provocaram a doença de Parkinson em macacos, induzida farmacologicamente pela substância 1-metil-4-fenil-1,2,3,6-tetraidropiridina. Após instalada e diagnosticada a doença de Parkinson, os cientistas inativaram o núcleo subtalâmico por meio de neurotoxina (ácido ibotênico). Os resultados mostraram que a inativação do núcleo subtalâmico ocasionava um incremento das habilidades motoras no membro contralateral ao núcleo estimulado, enquanto o membro ipsilateral não mostrava nenhuma melhora. Postulou-se que a ativação excessiva do núcleo subtalâmico tenha um papel muito importante na doença de Parkinson. Os efeitos positivos na doença de Parkinson provocados pela EEP no núcleo subtalâmico podem ter um efeito inibitório.

Maks et al. ${ }^{25}$ (2009) utilizaram EEP monopolar catódica no núcleo subtalâmico unilateralmente, com o objetivo de encontrar a área do núcleo que respondia com maior atividade tecidual. O estudo contou com dez participantes com diagnóstico de doença de Parkinson. Os resultados foram mostrados por meio de imagem por ressonância magnética e por tomografia computadorizada. Concluiu-se que a estimulação próxima à borda da região dorsal do núcleo subtalâmico respondia com maior atividade tecidual que a estimulação em outras regiões.

Bewernick et al. ${ }^{7}$ (2010) avaliaram a estimulação bilateral do núcleo accumbens para tratamento de depressão e anedonia. Participaram da pesquisa dez voluntários com quadro depressivo que não obtiveram boa resposta com o uso de fármaco- psico- e eletroconvulsoterapia. O formato da onda era quadrado com parâmetros em 90 us de período ativo do pulso, com $130 \mathrm{~Hz}$ de frequência e amplitude de $2 \mathrm{~V}$. O estímulo era constante durante uma semana. A partir da segunda semana, a amplitude da EEP foi incrementada de $2 \mathrm{~V}$ para $4 \mathrm{~V}$. Após quatro semanas, se os participantes não demonstrassem qualquer melhora, os parâmetros eram reajustados para: amplitude de $10 \mathrm{~V}$, frequência de 150 $\mathrm{Hz}$ e período ativo do pulso em $210 \mu \mathrm{s}$. Os efeitos adversos foram relacionados ao procedimento cirúrgico $\mathrm{e}$ restringiram-se a edema, disfagia e dor. Alterações dos parâmetros da EEP proporcionavam: eritema, aumento transitório da ansiedade e sudorese, além de alterações não relacionadas à EEP como gastrite e fratura de perna. Após seis meses de tratamento, imagens de tomografia por emissão de pósitrons mostraram redução metabólica das regiões: (1) córtex pré-frontal orbital, (2) região anterior (área 25 de Brodmann) ${ }^{24} \mathrm{e}$ (3) região posterior do giro cingulado, (4) tálamo e (5) núcleo caudado. Com resultados contrários às regiões citadas acima, o giro pré-central (área motora primária - 4 de Brodmann) ${ }^{24}$ mostrou um aumento do seu metabolismo. Os resultados mostraram que, após 12 meses de tratamento, cinco participantes tiveram redução de $50 \%$ na escala de depressão de Hamilton (0-62 pontos, quanto maior pior). Concluiu-se que a EEP possui efeitos positivos sobre a depressão e a anedonia.

Péron et al. ${ }^{31}$ (2010) avaliaram se a EEP aplicada ao núcleo subtalâmico bilateralmente para tratamento da doença de Parkinson prejudica o reconhecimento de 
alterações emocionais na fala (prosódia). Levantou-se a hipótese de que a EEP alteraria o metabolismo de glicose em estruturas límbicas como a da amígdala e da região orbital do lobo frontal. No estudo, participaram pacientes com doença de Parkinson em estágio pós-operatório do implante da EEP $(\mathrm{N}=21)$ e voluntários hígidos para o controle $(\mathrm{N}=21)$. Os parâmetros da EEP foram: forma de onda monofásica com eletrodo tetrapolar e com valores diferenciados entre o lado direito e esquerdo. Os testes foram realizados com palavras em prosódias distintas como raiva, medo, felicidade e tristeza. Os resultados foram inconclusivos, pois tanto antes quanto após a cirurgia não houve diferença estatística entre os participantes com doença de Parkinson. Houve diferença estatística entre os voluntários com doença de Parkinson e os voluntários hígidos, tanto antes quanto depois do procedimento cirúrgico.

Alberts et al. ${ }^{3}$ (2010) compararam os resultados do uso de EEP com localização dos eletrodos por meio de (1) parâmetros clínicos, por localização obtida por (2) modelagem computacional. A modelagem computacional estimava a região de maior volume de ativação tecidual na EEP bilateral dos núcleos subtalâmicos para o tratamento da doença de Parkinson. Foram avaliadas as performances das respostas motora e cognitiva geradas para EEP com diferentes ativações (1 e 2). A pesquisa consistiu de um estudo de caso que teve como participante um homem de 58 anos de idade com histórico de doença de Parkinson havia oito anos. Catorze meses antes da pesquisa, o participante havia passado por procedimento cirúrgico da EEP. Os parâmetros do estimulador elétrico foram configurados com valores distintos para cada lado, sendo o período ativo do pulso sempre $60 \mu \mathrm{s}$, amplitude de 2 a 3,2 V, e frequência de 130 a $185 \mathrm{~Hz}$. No lado esquerdo, os parâmetros clínicos tinham uma ativação mais ventral que os parâmetros da modelagem computacional, que foram mais dorsais. Um dos resultados importantes foi que para os (1) parâmetros clínicos, os testes que envolviam tarefas de força, os resultados foram similares tanto quando a EEP estava ligada como quando estava desligada, indicando supostamente uma resposta não motora das conexões do núcleo subtalâmico (região ventral medial do lado esquerdo). As tarefas cognitivas e motoras tiveram melhores resultados pelo posicionamento estimulado pela modelagem computacional, possivelmente pela ativação das regiões mais dorsais do núcleo subtalâmico.

Fontaine et al. ${ }^{14}(2010)$ avaliaram a aplicação da EEP no hipotálamo posterior para o tratamento da cefaleia em salva. Dez pacientes tiveram eletrodos posicionados no hipotálamo posteroinferior, onde o eletrodo era posicionado ipsilateralmente ao lado afetado. Com um ano de uso da EEP, cinco deles responderam positivamente ao tratamento, e a frequência semanal de ataques de cefaleia em salva foi $<50 \%$ comparando-se com o período pré-cirúrgico. Os outros cinco participantes foram inclusos como grupo controle. O posicionamento dos eletrodos foi distinto em relação à aderência intertalâmica. As regiões com maior eficiência durante a aplicação situam-se posteriormente ao hipotálamo. As estruturas que estavam localizadas a menos de $2 \mathrm{~mm}$ dessas regiões com maior eficiência foram: a substância periaquedutal (05/05), o núcleo rubro (05/04), o fascículo retroflexo (05/04), o fascículo dorsal longitudinal (05/03), o núcleo da alça lenticular (05/03), o fascículo longitudinal medial $(05 / 01)$ e a superfície medial do tálamo (05/01). Não houve diferença estatística entre os grupos que obtiveram efeitos positivos em comparação ao grupo controle. Concluiu-se que os efeitos positivos da EEP sobre a cefaleia em salva não está diretamente ligada à estimulação do hipotálamo posterior.

Ackermans et al. ${ }^{1}$ (2010) avaliaram em dois estudos de caso a influência da EEP na síndrome de Tourette em estudo longitudinal. O participante 1 tinha dez anos de implante e o participante 2, seis. A estimulação foi realizada na região medial do tálamo bilateralmente. Avaliaram-se a redução do tique, o estado de cognição e efeitos secundários. Para o participante 1 , a redução dos tiques foi de $90,1 \%$ nos primeiros cinco anos e de $92,6 \%$ ao final dos dez anos. Para o participante 2 , a redução dos tiques chegou a $82 \%$ durante os primeiros oito meses do tratamento e decaiu para $78 \%$ ao final de seis anos do início do tratamento. A fluência verbal manteve-se igual ao longo do tratamento para o participante 1 . $\mathrm{O}$ participante 2 teve uma redução da fluência verbal, da capacidade de aprendizado e apresentou um comportamento mais hostil com leve redução do quadro depressivo, mas ainda com quadro de difícil adaptação social.

Azoulay-Zyss et al. ${ }^{4}$ (2011) aplicaram a EEP bilateralmente no globo pálido interno em cinco pacientes com mioclonia-distonia, decorrente de deficiência genética da proteína $\varepsilon$-sarcoglicana. A escala de avaliação de distonia Burke-Fahn-Marsden (de 0 a 120) e a escala de avaliação de mioclonia unificada (de 0 a 288) foram aplicadas seis a nove meses e 15 a 18 meses após a cirurgia. A amplitude configurada inicialmente foi de $25 \%$ a $30 \%$ inferior ao limiar de efeitos adversos, sendo aumentada progressivamente nos primeiros três meses até se encontrar a amplitude ótima sem causar efeitos adversos. Os resultados médios dos voluntários para mioclonia chegaram a 30 antes da cirurgia e decaíram para 4,5 após os seis meses do processo cirúrgico.

Klein et al. ${ }^{19}$ (2011) avaliaram em modelo animal empregando ratos a estimulação do núcleo subtalâmico com eletrodos bipolares. Foi utilizado o marcador radioativo fluoro-D-glucose (FDG) com tomografia computadorizada por emissão de pósitrons para análise imaginológica, juntamente com análise histoquímica 
post mortem. Estudos de imagem mostraram aumento do metabolismo (1) no tronco cerebral, (2) no córtex cingulado ipsi e contralateral, (3) no núcleo meio-dorsal do tálamo, (4) no globo pálido e (5) no núcleo caudato ipsilaterais. Além disso, constatou-se diminuição da absorção do marcador FDG na (6) amígdala, (7) córtex entorinal e (8) córtex somatossensorial ipsi e contralaterais, na região do (9) hipocampo ispsilateral e (10) córtex pré-límbico contralateral. A transcrição da susbtância c-Fos é regulada em resposta a vários sinais extracelulares como fatores de crescimento. Resultados histoquímicos revelaram indução da c-Fos no (11) tronco cerebral ipsi e contralateral, (12) na amígdala, (13) no núcleo talâmico mediodorsal, (14) no globo pálido e (15) no córtex cingulado ipsilateralmente. Modelos animais são importantes para corroborar as alterações fisiológicas post mortem decorrentes da EEP.

\section{Conclusão}

A estimulação encefálica profunda, portanto, está sendo aplicada em patologias que acarretam alterações motoras e/ou psicológicas. Os resultados apresentados nos tratamentos realizados são predominantemente positivos, reduzindo ou descartando a utilização de medicação complementar. Dos parâmetros estimulatórios utilizados entre os anos de 2009 e 2010, destacam-se: amplitude de 1 a $10 \mathrm{~V}$, frequência de 130 a $185 \mathrm{~Hz}$, corrente de 50 a $300 \mu \mathrm{A}$ e período ativo do pulso de 60 a $220 \mu \mathrm{s}$. A estimulação encefálica profunda desponta como recurso promissor no tratamento de algumas doenças e substituição da terapia medicamentosa, mesmo sendo necessário procedimento cirúrgico concomitante para sua utilização, e abre a possibilidade de mais pesquisas para avaliar sua aplicação em novos campos.

\section{Agradecimentos}

Os autores agradecem à Coordenação de Aperfeiçoamento de Pessoal de Nível Superior (Capes) e Conselho Nacional de Desenvolvimento Científico e Tecnológico (CNPq) pelas bolsas concedidas para a realização deste trabalho.

\section{Referências}

1. Ackermans L, Duits A, Temel Y, Winogrodzka A, Peeters $F$, Beuls EAM, et al. Long-term outcome of thalamic deep brain stimulation in two patients with Tourette syndrome. J Neurol Neurosurg Psychiatry. 2010;81(10):1068-72.

2. Alam M, Schwabe K, Krauss JK. The pedunculopontine nucleus area: critical evaluation of interspecies differences relevant for its use as a target for deep brain stimulation. Brain. 2011;134(Pt1):11-23.

3. Alberts JL, Hallahan K, Thota A, Noecker AM, Vitek $\mathrm{JL}$, Mclntyre CC. Reducing cognitive-motor declines associated with bilateral subthalamic deep brain stimulation through computational modelling in a Parkinson's disease patient. J Neurol Neurosurg Psychiatry. 2010;81(10):1170.

4. Azoulay-Zyss J, Roze E, Welter ML, Navarro S, Yelnik J, Clot $F$, et al. Bilateral deep brain stimulation of the pallidum for myoclonus-dystonia due to \{varepsilon\}-sarcoglycan mutations: a pilot study. Arch Neurol. 2011;68(1):94-8.

5. Benabid AL, Chabardes S, Mitrofanis J, Pollak P. Deep brain stimulation of the subthalamic nucleus for the treatment of Parkinson's disease. Lancet Neurol. 2009;8(1):67-81.

6. Bergman H, Wichmann T, DeLong MR. Reversal of experimental parkinsonism by lesions of the subthalamic nucleus. Science. 1990;249(4975):1436-8.

7. Bewernick BH, Hurlemann R, Matusch A, Kayser S, Grubert C, Hadrysiewicz B, et al. Nucleus accumbens deep brain stimulation decreases ratings of depression and anxiety in treatment-resistant depression. Biol Psychiatry. 2010;67(2):110-6.

8. Borggraefe I, Mehrkens JH, Telegravciska M, Berweck S, Bötzel K, Heinen F. Bilateral pallidal stimulation in children and adolescents with primary generalized dystonia - report of six patients and literature-based analysis of predictive outcomes variables. Brain Dev. 2010;32(3):223-8.

9. Cools R, Miyakawa A, Sheridan M, D'Esposito M. Enhanced frontal function in Parkinson's disease. Brain. 2010;133(Pt 1):225-33.

10. DiLorenzo DJ, Bronzino JD. Neuroengineering. Boca Raton, FL: CRC Press; 2007.

11. Felten DL, Józefowicz RF. Atlas de neurociências humana de Netter. [Reimpressão]. Porto Alegre: Artmed; 2005.

12. Fisher R, Salanova V, Witt T, Worth R, Henry T, Gross R, et al. Electrical stimulation of the anterior nucleus of thalamus for treatment of refractory epilepsy. Epilepsia. 2010;51(5):899-908.

13. Follett KA, Weaver FM, Stern M, Hur K, Harris CL, Luo P, et al. Pallidal versus subthalamic deep-brain stimulation for Parkinson's disease. N Engl J Med. 2010;362(22):2077-91.

14. Fontaine $\mathrm{D}$, Lanteri-Minet $\mathrm{M}$, Ouchchane $\mathrm{L}$, Lazorthes $\mathrm{Y}$, Mertens $\mathrm{P}$, Blond $\mathrm{S}$, et al. Anatomical location of effective deep brain stimulation electrodes in chronic cluster headache. Brain. 2010;133:1214-23.

15. Gutman DA, Holtzheimer PE, Behrens TEJ, JohansenBerg H, Mayberg HS. A tractography analysis of two deep brain stimulation white matter targets for depression. Biol Psychiatry. 2009;65(4):276-82.

16. Haines DE. Neuroanatomy: an atlas of structures sections, and systems. 7th. Baltimore: Lippincott Williams \& Wilkins; 2008.

17. He B. Neural engineering. Dordrecht, The Netherlands: Kluwer/Plenum; 2005.

18. Inteligemcia. Primeira cirurgia para implantação de marcapasso recarregável é realizada no Hospital Alemão Oswaldo Cruz. 2011 [cited 21 out. 2011]. Disponível em: http:// www.inteligemcia.com.br/29642/2011/05/03/primeiracirurgia-para-implantacao-de-marca-passo-recarregavele-realizada-no-hospital-alemao-oswaldo-cruz/.

19. Klein J, Soto M, Pascau J, Günther L, Kupsch A, Desco $M$, et al. A novel approach to investigate neuronal network 
activity patterns affected by deep brain stimulation in rats. J Psychiatr Res. 2011;45(7):927-30.

20. Krack P, Hariz MI, Baunez C, Guridi J, Obeso JA. Deep brain stimulation: from neurology to psychiatry? Trends Neurosci. 2010;33(10):474-84.

21. Krueger-Beck E, Scheeren EM, Nogueira-Neto GN, Button VLdSN, Nohama P. Efeitos da estimulação elétrica funcional no controle neuromuscular artificial. Rev Neurocienc. 2011;19(3):530-41.

22. Lakhan SE, Callaway E. Deep brain stimulation for obsessive-compulsive disorder and treatment-resistant depression: systematic review. BMC Research Notes. 2010;3(1):60-8.

23. Lozano AM, Snyder BJ, Hamani C, Hutchison WD, Dostrovsky JO. Basal ganglia physiology and deep brain stimulation. Mov Disord. 2010;25(S1):S71-5.

24. Machado ABM. Neuroanatomia funcional. 2. ed. São Paulo: Atheneu; 2006.

25. Maks CB, Butson CR, Walter BL, Vitek JL, McIntyre CC. Deep brain stimulation activation volumes and their association with neurophysiological mapping and therapeutic outcomes. J Neurol Neurosurg Psychiatry. 2009;80(6):659-66.

26. Meneses MS, Follador FR, Arruda WO, Santos HL, Yonesawa D, Hunhevicz SC. Implantação estereotáxica de eletrodos profundos por ressonância magnética para cirurgia de epilepsia. Arq Neuro-Psiquiatr. 1999;57(3A):628-35.

27. Meneses MS, Teive HAG. Doença de Parkinson. 2. ed. Rio de Janeiro: Guanabara/Koogan; 2003.

28. Merrill DR, Bikson M, Jefferys JGR. Electrical stimulation of excitable tissue: design of efficacious and safe protocols. J Neurosci Methods. 2005;141(2):171-98.

29. Netter FH. Atlas de anatomia. 2. ed. Traduzido por Jackes Vissoky e Eduardo Cotecchia Ribeiro. Porto Alegre: Artmed; 2001.

30. Okun MS, Fernandez HH, Wu SS, Kirsch Darrow L, Bowers D, Bova F, et al. Cognition and mood in Parkinson's disease in subthalamic nucleus versus globus pallidus interna deep brain stimulation: the COMPARE trial. Ann Neurol. 2009;65(5):586-95.
31. Péron J, Grandjean D, Le Jeune F, Sauleau P, Haegelen C, Drapier D, et al. Recognition of emotional prosody is altered after subthalamic nucleus deep brain stimulation in Parkinson's disease. Neuropsychologia. 2010;48(4):1053-62.

32. Pinault D. Deep Brain Stimulation. Eur J Neurosci. 2010;32(7):1-7.

33. Rahman M, Murad GJA, Mocco J. Early history of the stereotactic apparatus in neurosurgery. Neurosurg Focus. 2009;27(3):1-5

34. Rossi C, Marino Júnior R. Epilepsia temporal. Arq NeuroPsiquiatr. 1988;46(1):38-48.

35. Rouaud T, Lardeux S, Panayotis N, Paleressompoulle D, Cador M, Baunez $\mathrm{C}$. Reducing the desire for cocaine with subthalamic nucleus deep brain stimulation. Proc Natl Acad Sci. 2010;107(3):1196-200.

36. Schwalb JM, Hamani C. The history and future of deep brain stimulation. Neurotherapeutics. 2008;5(1):3-13.

37. Sen AN, Campbell PG, Yadla S, Jallo J, Sharan AD. Deep brain stimulation in the management of disorders of consciousness: a review of physiology, previous reports, and ethical considerations. Neurosurg Focus. 2010;29(2):14-6.

38. Weaver FM, Follett K, Stern M, Hur K, Harris C, Marks WJ $\mathrm{J}$, et al. Bilateral deep brain stimulation vs best medical therapy for patients with advanced Parkinson disease: a randomized controlled trial. JAMA. 2009;301(1):63-73.

39. Wojtecki L, Vesper J, Schnitzler A. Interleaving programming of subthalamic deep brain stimulation to reduce side effects with good motor outcome in a patient with Parkinson's disease. Parkinsonism Relat Disord. 2011;17(4):293-4.

\section{Endereço para correspondência}

Eddy Krueger

Universidade Tecnológica Federal do Paraná (UTFPR) - Laboratório de

Engenharia de Reabilitação-CPGEI

Av. Sete de Setembro, 3165, Rebouças

80230-901 - Curitiba, $P R$

Telefone: (55 41) 3310-4489

E-mail: kruegereddy@gmail.com 